\title{
Extracellular Potentials from Active Myelinated Fibers Inside Insulated and Noninsulated Peripheral Nerve
}

\author{
Jan H. Meier, Wim L. C. Rutten,* Member, IEEE, and Herman B. K. Boom, Senior Member, IEEE
}

\begin{abstract}
A model is presented that calculates the singlefiber extracellular field and action potential (ap) of an active myelinated nerve fiber placed centrally or eccentrically inside a nerve with a cylindrical geometry, representing essentially a onefascicle nerve. This one-fascicle nerve has the dimensions and conductivities of the rat peroneal nerve branch. The results show a wide variety of wave shapes to be measured, depending on the position of the intraneural electrode with respect to the fiber axis and to the nodes of Ranvier and depending on the presence of an isolating cuff around the nerve. Action potential shapes may range from the "classical" quasi-biphasic one, to more triphasic, or even more complicated in the case of a short insulating cuff being present around the nerve. In the latter case, when measured bipolarly, ap-wave shapes become almost monophasic.
\end{abstract}

Index Terms -Action potential (ap) recording, extracellular ap modeling, functional electric stimulation and recording, nerve cuff, selective recording.

\section{INTRODUCTION}

$\mathbf{I}$ $\mathrm{N}$ neurophysiological and rehabilitation research there is an increasing interest in techniques to provide information to and extract information from peripheral nerve (for example [1], [2], [6]-[8], [12], and [14]). The optimal device for these techniques would allow for the selective stimulation of the nerve fibers as well as the selective recording of their activity.

It has been shown that in case of electrical stimulation the highest degree of selectivity can be achieved with dense three-dimensional "lattices" of electrodes, placed inside the fascicles [9]-[11], [18], [19]. These intraneural stimulation electrodes, however, can also be used to monitor nerve signals, for example, to be used in future closed-loop control systems for rehabilitation.

In this respect, it is of interest to examine by modeling whether selective recording in nerve is possible, and how electrode and fiber position, tissue inhomogeneities, and anisotropies influence ap-wave shape and amplitude. Also, for

Manuscript received June 26, 1997; revised March 3, 1998. Asterisk indicates corresponding author.

J. H. Meier was with the Biomedical Engineering Department, Faculty of Electrical Engineering/Institute for Biomedical Technology, University of Twente, 7500 AE Enschede, the Netherlands. He is now with the Biotronik/University Erlangen-Nurnberg, D-91052 Erlangen, Germany.

*W. L. C. Rutten is with the Biomedical Engineering Department, Faculty of Electrical Engineering/Institute for Biomedical Technology, University of Twente, P.O. Box 217, 7500 AE Enschede, the Netherlands.

H. B. K. Boom is with the Biomedical Engineering Department, Faculty of Electrical Engineering/Institute for Biomedical Technology, University of Twente, 7500 AE Enschede, the Netherlands.

Publisher Item Identifier S 0018-9294(98)05899-6. proper processing the signals (monopolar or bipolar recordings, filter settings, spatial averaging) it is of importance to know how the ap-wave shape and amplitude vary with position of the electrode and conductivity of surrounding media.

A typical fascicle has a diameter of $0.5 \mathrm{~mm}$, and the motor fibers within have typical diameters of 5-20 $\mu \mathrm{m}$, with corresponding node (of Ranvier) spacing of $0.5-2 \mathrm{~mm}$. The number of motor fibers may be up to several hundreds. With respect to selectivity of recording, these dimensions and numbers readily lead to the expectation that there will be a large influence of the distance of the electrode to an active fiber and of the distance to the nodes of Ranvier. This is mainly due to the large ratio of node spacing to fiber diameter (in the order of 100), implying that an electrode has to be in the immediate neighborhood (tens of micrometers) of a fiber's node to pick up its signal distinctly from that of neighboring fibers.

A number of studies has been done earlier on calculating action potentials (ap's) from myelinated nerve fibers [5], [8], [13], [15], but none of these has considered the combined effect of eccentricity of sources and the inhomogeneities and anisotropic conductivities of the various tissues involved, on ap-wave shapes.

This paper deals with the (selective) recording of nerve fiber activity with intrafascicular electrodes. An algorithm is developed for the calculation of the extracellular potential field of an active myelinated nerve fiber inside a fiber bundle and an application study is shown in which special interest is given to the conductivity of the medium that surrounds the nerve and to the radial position of the active nerve fiber. The calculation of single fiber ap's to be measured with an intrafascicular electrode is presented and it is shown how these signals depend on the position of the electrode relative to the fiber and its active nodes. Also, the effect of an insulating cuff around the nerve is calculated.

The results give clues for the amount of selectivity that can be reached with multielectrode device configurations.

\section{METHODS}

\section{A. Calculation of the Extracellular Potential Field of an Active Nerve Fiber}

For the fiber bundle and its surroundings a simplified conductor model is used and an analytical expression is derived for the potential field of a point current source placed 


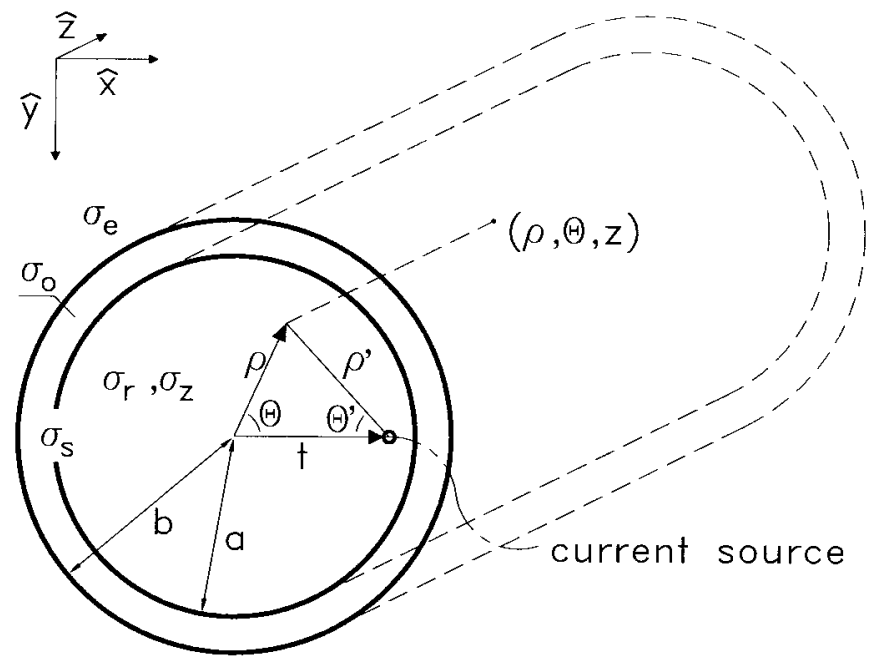

Fig. 1. Model of the nerve fascicle, with perineural sheath labeled " $s$," epineural space "o," and external surroundings labeled "e." For a detailed description, see the text.

TABLE I

PARAMETER VALUES

\begin{tabular}{|c|c|}
\hline $\begin{aligned} \sigma_{\rho} & =0.1 \Omega^{-1} \mathrm{~m}^{-1} \\
\sigma_{z} & =0.5 \Omega^{-1} \mathrm{~m}^{-1} \\
\sigma_{s} & =2000 \Omega^{-1} \mathrm{~m}^{-1} \\
\sigma_{o} & =0.1 \Omega^{-1} \mathrm{~m}^{-1} \\
\sigma_{e} & =10 / 0.1 / 0.001 \Omega^{-1} \mathrm{~m}^{-1} \\
a & =250 \mu \mathrm{m} \\
b & =320 \mu \mathrm{m} \\
r & =200 \mu \mathrm{m} \\
\lambda & =1000 \mu \mathrm{m} \\
v & =57.1 \mathrm{~m} / \mathrm{s}\end{aligned}$ & $\begin{array}{l}\text { conductivity of the fascicle in } \\
\text { radial and longitudinal direction } \\
\text { conductivity of the perineural sheath } \\
\text { conductivity of the epineurium } \\
\text { conductivity of the extraneurium } \\
\text { radius of the fascicle } \\
\text { radius of the nerve } \\
\text { radial position of the fiber } \\
\text { internode length } \\
\text { propagation velocity }\end{array}$ \\
\hline
\end{tabular}

arbitrarily inside this bundle. The active nerve fiber is modeled as a number of point current sources and its potential field is calculated as a linear summation of the fields of these separate sources.

1) The Nerve: A nerve is modeled that contains only one fascicle. It is idealized as two coaxial, homogeneous, infinitely extending cylinders (Fig. 1). The inner cylinder represents the fascicle and has a radius $a$, a radial conductivity $\sigma_{\rho}$ and a longitudinal conductivity $\sigma_{z}$. The outer cylindrical cell represents the epineurium and has a radius $b$ and an isotropic conductivity $\sigma_{o}$. Between the cylinders a sheath, with sheet conductivity $\sigma_{s}$, represents the thin perineurium. The nerve is immersed in a homogeneous and infinite medium with conductivity $\sigma_{e}$. The Appendix gives the calculation of the potential field due to a point current source placed eccentrically inside the inner cylinder.

The conductivities of the different tissues are given realistic values (for a detailed discussion on the conductivities; see [9]). The radii of the fascicle and the epineurium are given values as measured in histological sections of rat peroneal nerve. Table I lists the parameter values chosen.

2) The Active Nerve Fiber: The active fiber is simulated as an array of active point current sources along the fiber bundle (Fig. 2). Each source represents a node of Ranvier. In the simulation results shown in this paper, a nodal distance $\lambda$ of $1000 \mu \mathrm{m}$ is chosen. Current values correspond to the nodal

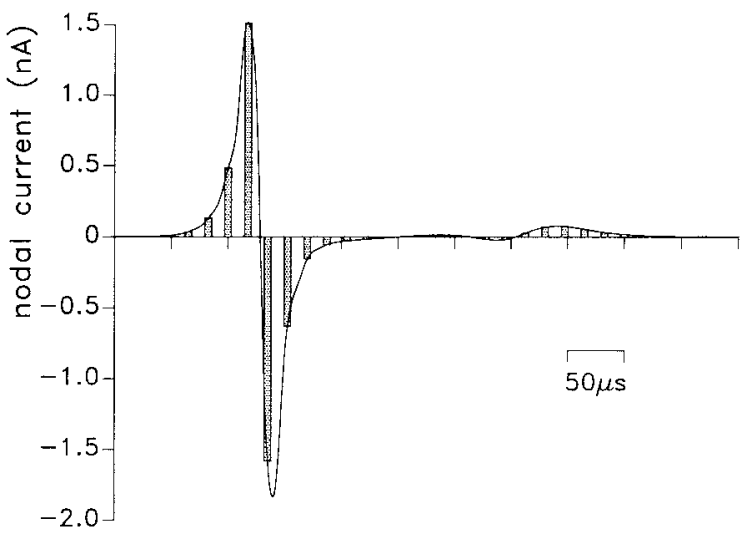

Fig. 2. The continuous line represents the nodal current as a function of time and the bars represent the currents of the active nodes at one instant of time as used in the simulations. Each bar corresponds to one node. The height of the bar indicates the nodal current.

currents of an active fiber of $10-\mu \mathrm{m}$ diameter at one instant of time. These nodal currents are calculated using the simulation model for myelinated nerve fibers developed by Struijk et al. [16], in which the membrane currents of a myelinated nerve fiber are calculated using the membrane ionic channel kinetics (Hodgkin-Huxley formulation) as described by Chiu et al. [3] These kinetics are valid at a temperature of $14^{\circ} \mathrm{C}$. The authors adopted the corrections of Struijk et al. [16] for a temperature of $37^{\circ} \mathrm{C}$.

\section{B. Calculation of the Signals to be Measured with Intrafascicular Electrodes}

The potential $\varphi$ at a point electrode at position $\left(x_{e}, y_{e}, z_{e}\right)$ due to $N$ active nodes of Ranvier can be written as

$$
\varphi\left(x_{e}, y_{e}, z_{e}, t\right)=\sum_{j=1}^{N}\left\{\psi_{x_{e}, y_{e}, z_{e}}\left(x_{j}, y_{j}, z_{j}\right) I_{j}(t)\right\}
$$

where $\left(x_{j}, y_{j}, z_{j}\right)$ and $I_{j}(t)$ are the position and the current of the node indexed by $j$ and $\psi$ is the lead field of the electrode. [According to the reciprocity principle the lead field is equal to the potential field that would be generated by a point source at position $\left(x_{e}, y_{e}, z_{e}\right)$ divided by the source current $I_{e}$. It can, thus, be calculated with the same equations as used to calculate the potential fields, see the Appendix.]

If only one nerve fiber is active, (1) can be rewritten as

$$
\begin{aligned}
& \varphi\left(x_{e}, y_{e}, z_{e}, t\right) \\
& \quad=\sum_{n=-\infty}^{\infty}\left\{\psi_{x_{e}, y_{e}, z_{e}}\left(x_{o}, y_{o}, z_{o}+n \lambda\right) I_{o}\left(t-\frac{n \lambda}{v}\right)\right\}
\end{aligned}
$$

or

$$
\begin{aligned}
& \varphi\left(x_{e}, y_{e}, z_{e}, t\right) \\
& \quad=\sum_{n=-\infty}^{\infty}\left\{\psi_{x_{o}, y_{o}, z_{o}}\left(x_{e}, y_{e}, z_{e}-n \lambda\right) I_{o}\left(t-\frac{n \lambda}{v}\right)\right\}
\end{aligned}
$$

where $\left(x_{o}, y_{0}, z_{0}\right)$ is the position of the nearest node of the active fiber, $I_{o}(t)$ the current at this node (see Fig. 2), $v$ the propagation velocity and $\lambda$ the internodal distance.

\section{Calculation of the Effect of a Nerve Cuff}

At the recording of nerve signals, the nerve is often insulated as much as possible to increase the signal amplitude. Common 


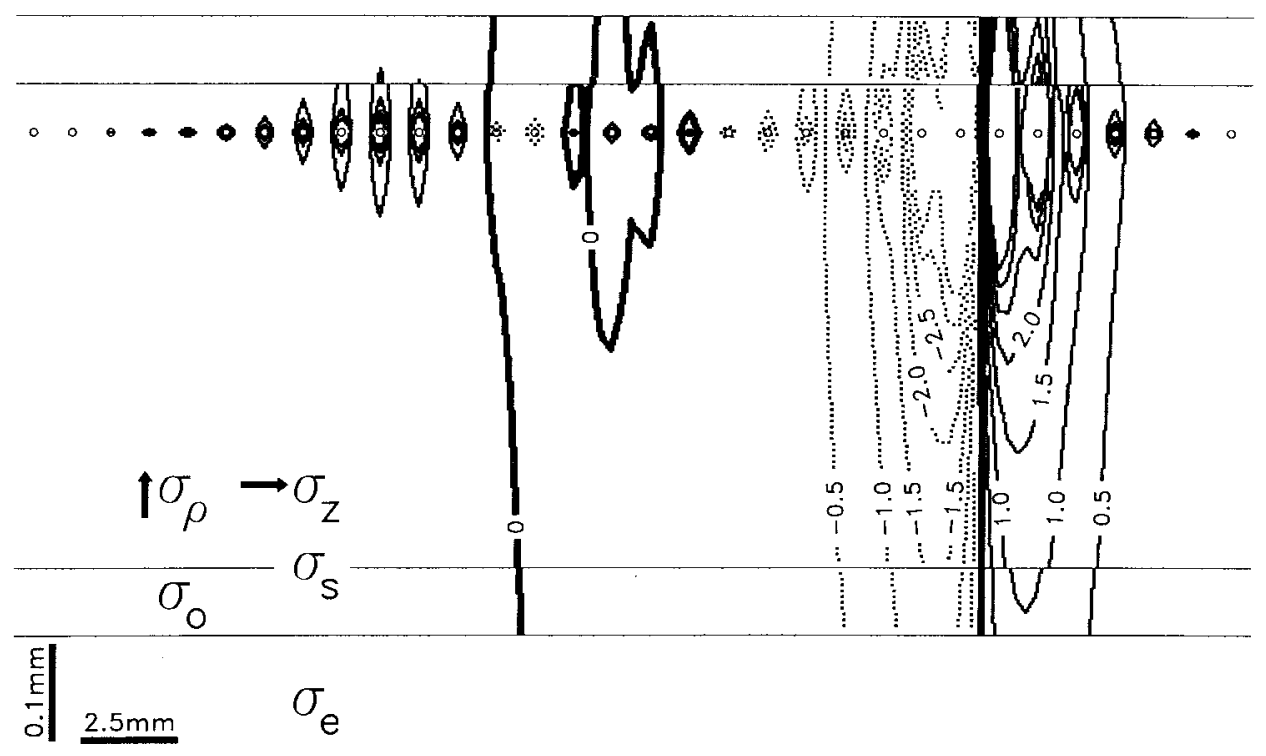

Fig. 3. An iso-potential contour plot of the extracellular potential field generated by an eccentric active nerve fiber, in a plane through the axes of the nerve and the fiber. The nerve is embedded in a medium with conductivity $\sigma_{e}=0.1 \Omega^{-1} \mathrm{~m}^{-1}$. The dotted/continuous lines are iso-contours corresponding to negative/positive iso-values, respectively, the thick drawn lines correspond to iso-value zero. Isopotential lines are drawn for $\varphi=0, \pm 0.5, \pm 1, \pm 1.5, \pm 2, \pm 4,-6$, and $-8 \mu \mathrm{V}$. Each circle denotes the position of a node. For the parameter values used see Table I.

procedures are the application of an insulating nerve cuff, the suspension of the nerve in air and/or paraffin oil, and the use of a tightly fitting nerve chamber (e.g., [1], [2], [7], and [14]). The simulation results for an insulated nerve cannot be applied straightforwardly to such a configuration when the opposite ends of the fiber bundle are electrically shunted (e.g., by external tissue or fluid around the insulation). Part of the currents generated by an active fiber will run outside the nerve tissue and, consequently, the current pattern and the potential field inside the fiber bundle will be changed.

Consider a nerve surrounded by a nerve cuff of length $L$. The origin is placed at the center of the cuff and an active nerve fiber is positioned along the axis of the fiber bundle. If the nerve and cuff were completely suspended in air, the potential field $\varphi(x, y, z, t)$ inside the fiber bundle will be that of an insulated nerve and, thus, at any time $t$, is dependent only on one parameter $z-v t$

$$
\varphi(x, y, z, t) \approx \xi(z-v t)
$$

where $v$ is the propagation velocity of the active fiber.

Upon immersion of the cuff and the nerve in a conducting tissue or fluid the potential field, $\varphi(x, y, z, t)$ changes to a new field $\varphi^{\prime}(x, y, z, t)$. Since there is generally a large volume of well-conducting surrounding fluid (or tissue) the total resistance outside the cuff will be negligible compared to the longitudinal resistance inside the cuff. So, the cuff ends have the same, zero, potential

$$
\begin{aligned}
\left.\varphi^{\prime}(x, y,-L / 2, t)\right|_{x^{2}+y^{2}=b^{2}} & =0 \\
\left.\varphi^{\prime}(x, y, L / 2, t)\right|_{x^{2}+y^{2}=b^{2}} & =0 .
\end{aligned}
$$

Inside the nerve cuff no extra current sources are added and, therefore, $\varphi(x, y, z, t)$ is still a singular solution of the Laplace equation. The regular solution to be added is a homogeneous longitudinal field along the $z$-direction that can vary in time: $\varphi_{\text {reg }}^{\prime}(x, y, z, t)=C_{1}(t) z+C_{2}(t)$. Addition of this solution and application of the new boundary conditions leads to the new solution $\varphi^{\prime}(x, y, z, t)$

$$
\begin{aligned}
\varphi^{\prime}(x, y, z, t)= & \xi(z-v t)+(z / L-1 / 2) \xi(-L / 2, t) \\
& -(z / L+1 / 2) \xi(L / 2, t) .
\end{aligned}
$$

\section{RESULTS}

\section{A. The Extracellular Potential Field of an Active Nerve Fiber within a Noninsulated Nerve}

Fig. 3 shows an iso-potential contour plot of the extracellular potential field of an active nerve fiber inside a nerve that is surrounded by tissue with normal conductivity (e.g., muscle tissue). The ap travels from the left to the right. On the righthand side of the plot the first phase of the transmembrane ap arrives. The nodal currents in this region are strongly positive and so are the values of the extracellular potential field. More toward the middle of the plot, the second phase of the transmembrane ap is present, and the field has negative values. On the left-hand side, the third phase of the ap results in small, positive field values.

In the middle of the plot, the potential field predominantly has negative values, due to the large second phase of the ap. However, around a few nodes within this area the potential field has small positive values since the currents of these nodes are positive (although small) and dominate the field in the neighborhood of these nodes.

\section{B. The Extracellular Potential Field within an Insulated Nerve}

Fig. 4 shows the nerve being placed inside a medium with low conductivity. As has been predicted [5], [9], [13], the potential values within this (nearly) insulated nerve are considerably higher than in the previous situation. Fig. 4 shows the radial electrical fields near the active fiber to be small compared to the longitudinal electrical fields; the transverse planes are nearly iso-potential planes. The signals 


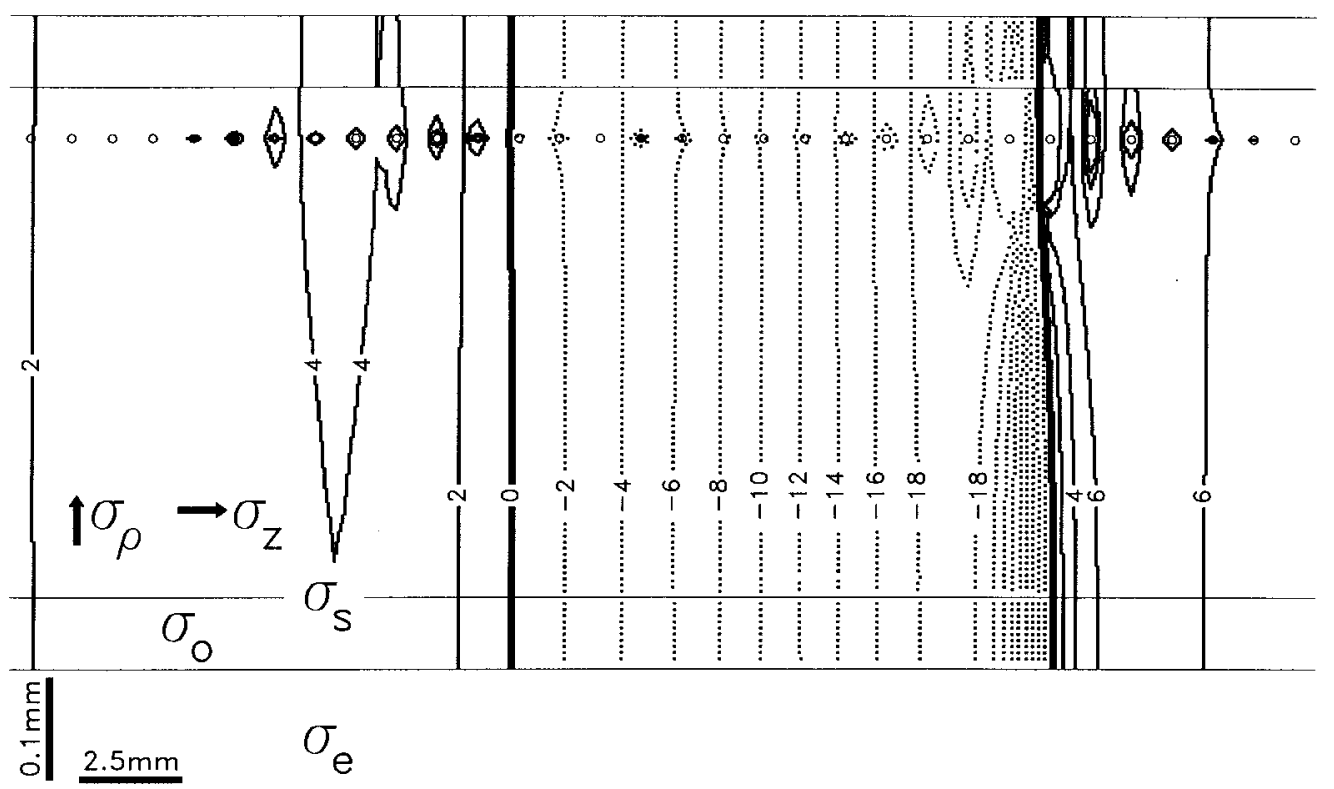

Fig. 4. An iso-potential contour plot of the potential field generated by an active nerve fiber inside a medium with conductivity $\sigma_{e}=0.001 \Omega^{-1} \mathrm{~m}^{-1}$. Iso-values are in $\mu \mathrm{V}$. For the parameter values used see Table I.

measured with an intrafascicular electrode will thus be almost independent of the radial positions of the active fibers and the electrode. Only, close to an active node the potential measured has a radial dependency on node and electrode position.

The reason for the transverse iso-potential planes can be found in the fact that at a larger scale an insulated nerve acts as an one-dimensional conductor, a "wire." From moderate distances (in the order of 50-100 $\mu \mathrm{m}$ ) from each single active node of Ranvier on, its currents are forced to flow parallel to the $z$-direction as the insulation does not allow current to cross the nerve boundary. As a result, the corresponding electrical field becomes constant and homogeneous.

\section{The Single-Fiber Signals Measured with Intrafascicular Electrodes}

Equation (2) is an expression for the single-fiber ap that is measured at position $\left(x_{e}, y_{e}, z_{e}\right)$. This signal is computed for a central active nerve fiber within nerves, surrounded by media of various conductivities. The position of the electrode is varied as shown in Fig. 5. The corresponding single fiber signals are plotted in Fig. 6.

The fiber signals calculated for extraneural conductivities of 10 and $0.1 \Omega^{-1} \mathrm{~m}^{-1}$ are predominantly biphasic. Their shapes are very similar to the shape of the nodal current as shown in Fig. 2. Their amplitudes decrease not only with the radial distance between fiber and electrode but also with the longitudinal distance between the electrode and the nearest node of Ranvier. Also, most notable at larger distances from the nodes of Ranvier, the amplitudes increase with a decreasing extraneural conductivity.

For a low extraneural conductivity of $0.001 \Omega^{-1} \mathrm{~m}^{-1}$, the fiber signals not only have increased further but also became triphasic. Their shape no longer resembles that of the nodal current. This is because the field due to the current of the nearest node no longer dominates. Each active node generates a homogeneous field throughout the whole bundle

$$
a-l: \text { electrodes }
$$

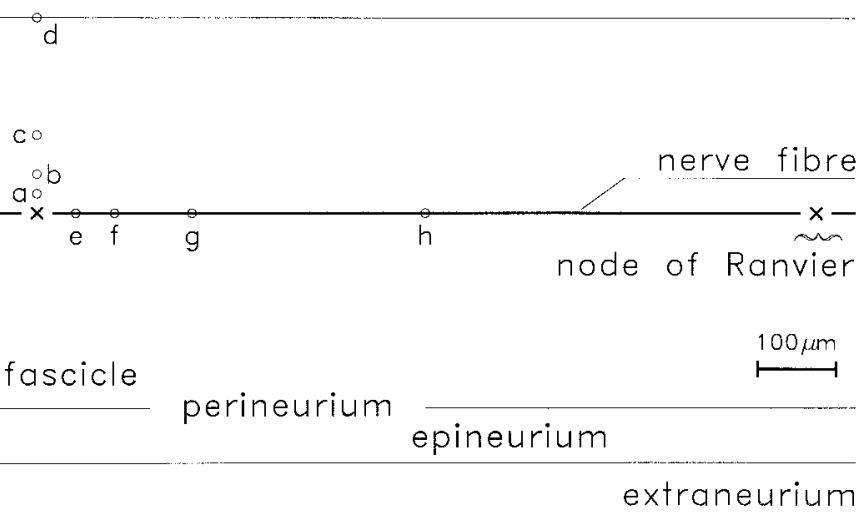

Fig. 5. The positions of the electrodes and the nerve fiber as used to calculate the single-fiber signals shown in Fig. 6 .

and, therefore, contributes to the signals measured at any place within. The signals are also strongly rippled which reflects the saltatory conduction of the ap. For a node of Ranvier, the transition from large positive currents to large negative currents (from phase one to phase two, Fig. 2) takes less time then the ap to travel one nodal distance. The phase transition "jumps" from node to node and due to the insulation its effect is seen on the fiber signal even if the recording electrode is at a large distance.

\section{The Effect of a Nerve Cuff on the Single-Fiber Signals}

In Fig. 7, a nerve is shown that is surrounded by a nerve cuff. The fiber bundle contains an active fiber with a node of Ranvier positioned at the center at the cuff. Near this node, an electrode is placed at a position as indicated by "c" in Fig. 5. Also shown are two electrodes, placed near the two neighboring nodes, to measure bipolar signals. The various parameter 


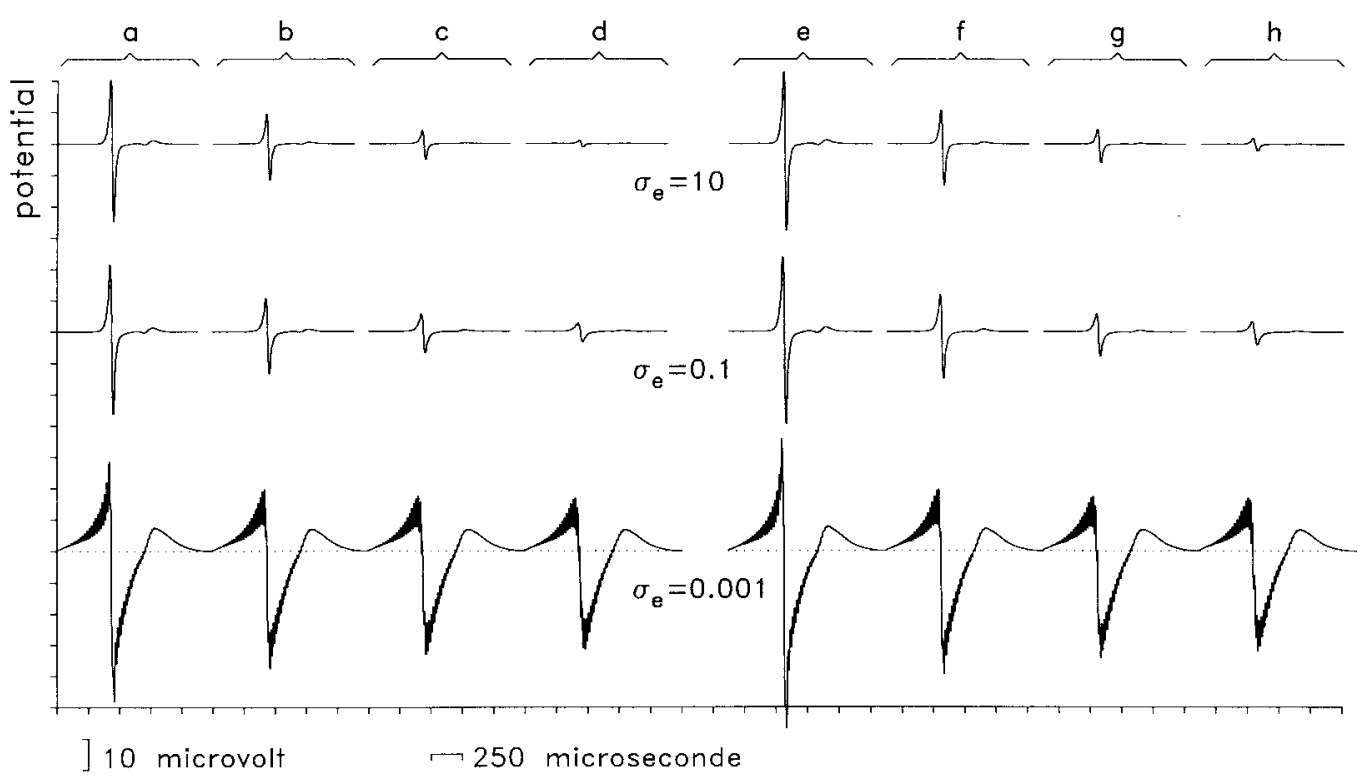

Fig. 6. The signals of a central nerve fiber as calculated for various values of the extraneural conductivity for the electrode positions shown in Fig. 5 (the lowest row, i.e., the "insulating" case, can be interpreted as for an infinite insulating cuff around the nerve).

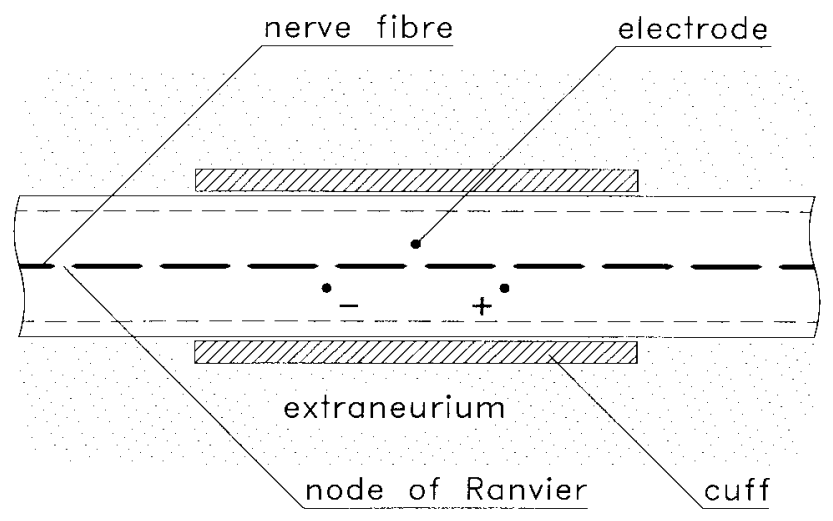

Fig. 7. A schematic view of a nerve surrounded by a nerve cuff of finite length. Three electrodes are placed inside the fiber bundle to measure the signal of a central nerve fiber. The whole system is embedded in a homogeneous conductive medium; the extraneurium.

values are equal to those of Fig. 4. The signals to be measured with the electrodes are shown in Fig. 8 for various cuff lengths.

The monopolar fiber signal calculated for a relatively long cuff of $40 \mathrm{~mm}$ is relatively complex and contains five phases. For shorter cuffs the number of phases decreases until the signal is predominantly biphasic.

The bipolarly measured fiber signals look almost monophasic, as the triphasic waveform has a strong second phase, flanked by two small phases of opposite sign. The duration of these small phases decreases with decreasing cuff length. For both monopolar and tripolar recording the signals amplitude decrease with cuff lengths below $10 \mathrm{~mm}$.

\section{DISCUSSION}

Models for calculating the extracellular potential field of an active myelinated fiber have been developed before but, for several reasons, these models fall short for our applications.

Stephanova et al. [15] simulated the active process along the membrane of a myelinated nerve fiber, embedded in an infinite homogeneous volume conductor, and calculated the corresponding extracellular potentials. The inhomogeneous geometrical structure of the nerve and its surroundings were not modeled.

Stein and Oguztöreli [13] derived an analytical expression for the extracellular potential field of a central active fiber inside a cylindrical homogeneous fiber bundle that was surrounded by an insulating cuff. In calculating the potential field, an extracellular membrane potential distribution $\varphi(z)$ was imposed at the surface of the active fiber. This nerve fiber was looked at as a voltage source, although a current source would be closer to reality.

Ganapathy and Clark [5] derived an expression similar to Stein and Oguztöreli, but at the surface of the active fiber they imposed a transmembrane current $i_{m}(z)$ as calculated using an equivalent circuit model for a representative myelinated nerve fiber.

The latter model comes closest to that presented in this paper. The present model has the refinements that 1) the tissues of the nerve (epineurium, perineurium, and fascicle) and its surroundings can be modeled separately, 2) the active fiber can be placed eccentrically, and 3) the fiber source is a distributed array of nodal current sources.

In the computations of Ganapathy and Clark [5], $i_{m}(z)$ had to be digitized in the $z$-domain. To span the whole active region a step size of $\Delta z=200 \mu \mathrm{m}$ was used. This step size is, however, much larger than the length of a node of Ranvier. Since, in reality, the membrane current is strongly peaked at these nodes, no accurate description could be achieved for the potential field in the neighborhood of the nodes. In the present model the primary source field (see Appendix) does not have to be digitized. In the neighborhood of a node, this field dominates and therefore a higher accuracy is achieved.

In the present model, the authors have assumed that the action currents of a nerve fiber are produced at the nodes 


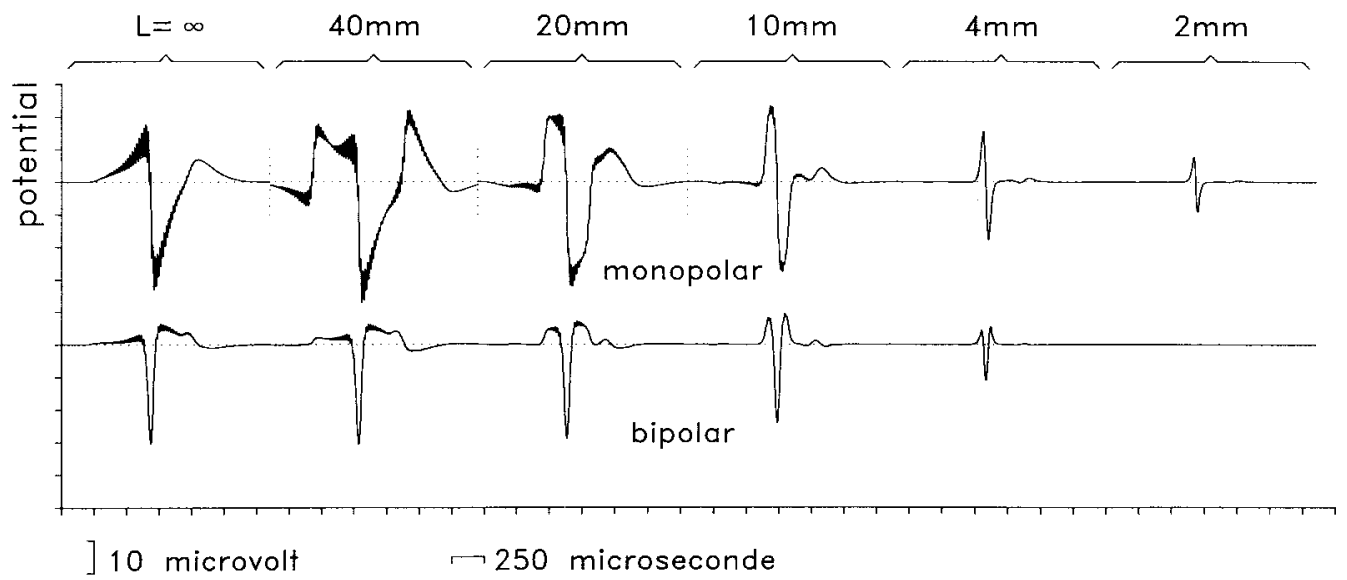

Fig. 8. The signal of a central nerve fiber for a monopolar intrafascicular electrode and for two differential intrafascicular electrodes 2 mm $(=2 \lambda)$ apart, at positions as indicated by "c" in Fig. 5. The nerve is surrounded by nerve cuffs with lengths of $\infty$, 40, 20, 10, 4, and 2 mm. The opposite ends of the cuffs are externally shunted. The electrode configurations are placed at the center of the cuff.

of Ranvier. The (capacitive) currents that flow through the myelin sheath were neglected since they are relatively small (see, e.g., Tasaki [17]).

For a noninsulated nerve it is shown that the amplitude of a fiber signal progressively increases with a decreasing distance between the recording electrode and the nearest node of Ranvier of the active nerve fiber. Therefore, the electrode can be said to be selective for those fibers which have a node within a close range.

The optimal situation for a selective multielectrode device would be an one-to-one correspondence between nodes and electrodes, i.e., when each electrode is close to exactly one node of Ranvier and vice versa. To approximate this situation an electrode density would be needed that is at least equal to the nodal density $N$ [4].

Assuming an average fiber diameter $(d)$ of $10 \mu \mathrm{m}$ and an average internodal length $(\lambda)$ of $1000 \mu \mathrm{m}$, and assuming that the fibers occupy half the space inside the fiber bundle, one finds $N_{0} \approx 2 /\left(\pi d^{2} \lambda\right) \approx 7 \cdot 10^{12}$ nodes $/ \mathrm{m}^{3}$. For a cubic grid of electrodes this implies a corresponding electrode spacing of $50 \mu \mathrm{m}$.

For lower electrode densities, each electrode will monitor the activity of a number of nerve fibers. The signals from different fibers on the same electrode then may be discriminated by their shape and their amplitude. This approach fails progressively with increasing electrode spacing when moredistant fibers elicit signals with increasingly similar shape and amplitude (Fig. 6, [4]). Calculations on the discriminating ability of electrodes when multiple nearby fibers are active have been presented elsewhere [19].

If the ap of an active fiber is measured with three or more monopolar electrodes, theoretically its position can be determined from the shape and amplitude of the corresponding signals (Fig. 6). This increases the ability to discriminate the activity of the separate fibers.

It was shown that if a nerve is insulated, the transverse planes become practically iso-potential planes. Only in those applications where fiber selectivity is not required is this insulation recommended, because it increases the signals mea- sured and makes them independent on the radial position of the electrode. One has to be very careful though in the interpretation of the shape and size of the fiber signals seen because they depend strongly on the length of the insulated part of the nerve (Fig. 8).

\section{APPENDIX \\ The Potential Field of a Point CuRrent Source IN THE Nerve Model}

A point current source at position $(t, 0,0)$ inside a fascicle (see Fig. 1) induces a potential field inside this fascicle that can be written as a superposition of a primary field $\varphi_{\text {prim }}$ and a secondary field $\varphi_{\text {sec }}$. The primary field is the field induced by the current source as if this source was placed in an infinite homogeneous medium. The secondary field represents the modifications of the field due to the inhomogeneities

$$
\begin{aligned}
\varphi_{i} & =\varphi_{\text {prim }}+\varphi_{\text {sec }} \\
& =\frac{I}{4 \pi \sqrt{\sigma_{\rho} \sigma_{z}} \sqrt{(x-t)^{2}+y^{2}+z^{2} \sigma_{\rho} / \sigma_{z}}}+\varphi_{\text {sec }} .
\end{aligned}
$$

Apart from the point current source, no extra sources are created. Therefore, the secondary field $\varphi_{\text {sec }}$, the field in the epineurium $\varphi_{0}$, and the field in the nerve surrounding medium $\varphi_{e}$, have to fulfill the equation of continuity

$$
\bar{\nabla} \cdot \bar{J}=0
$$

For isotropic conductive media this results in the Laplace equation. For the intrafascicular field, a proper coordinate transformation is needed

$$
x^{*}=\sqrt{\sigma_{z} / \sigma_{\rho}} x \quad y^{*}=\sqrt{\sigma_{z} / \sigma_{\rho}} y \quad z^{*}=z .
$$

We find

$$
\frac{\delta^{2} \varphi_{\mathrm{sec}}}{\left(\delta x^{*}\right)^{2}}+\frac{\delta^{2} \varphi_{\mathrm{sec}}}{\left(\delta y^{*}\right)^{2}}+\frac{\delta^{2} \varphi_{\mathrm{sec}}}{\left(\delta z^{*}\right)^{2}}=\Delta^{*} \varphi_{\mathrm{sec}}=\Delta \varphi_{o}=\Delta \varphi_{e}=0
$$


It can be shown that using a cylindrical coordinate system $\quad(\rho<t)$

$$
\begin{aligned}
\rho & =\sqrt{x^{2}+y^{2}} & & \\
\theta & =\arctan (-y / x) & & x>0 \\
& =\arctan (y / x) & & x<0 \\
& =\pi / 2 & & x=0, \quad y \leq 0 \\
& =3 \pi / 2 & & x=0, \quad y>0
\end{aligned}
$$

which is used in the general solution of the Laplace equation (for the $z$-direction the formulation will be in the frequency domain)

$$
\begin{aligned}
\varphi=\int_{-\infty}^{\infty}[ & \sum_{n=0}^{\infty}\left(A(n, k) I_{n}(k \rho)\right. \\
& \left.+B(n, k) K_{n}(k \rho)\right) \cos (n \theta)+\sum_{n=1}^{\infty}\left(C(n, k) I_{n}(k \rho)\right. \\
& \left.\left.+D(n, k) K_{n}(k \rho)\right) \sin (n \theta)\right] e^{i k z} d k
\end{aligned}
$$

where $I_{n}(k r)$ and $K_{n}(k r)$ are the modified Bessel functions of the first- and second-order. Using

$$
\frac{1}{\sqrt{\rho^{2}+z^{2}}}=\frac{1}{\pi} \int_{-\infty}^{\infty} K_{0}(k \rho) e^{i k z} d k
$$

and the Bessel function relations

$$
\begin{aligned}
& (\rho<t) \\
& K_{n}\left(k \rho^{\prime}\right) \cos \left(n \theta^{\prime}\right) \\
& =K_{n}(k t) I_{0}(k \rho)+\sum_{m=1}^{\infty}\left[K_{n+m}(k t)+K_{n-m}(k t)\right] \\
& \quad \cdot I_{m}(k \rho) \cos (m \theta) \\
& (\rho>t) \\
& K_{n}\left(k \rho^{\prime}\right) \cos \left(n \theta^{\prime}\right) \\
& =I_{n}(k t) K_{0}(k \rho)+\sum_{m=1}^{\infty}\left[I_{n+m}(k t)+I_{n-m}(k t)\right] \\
& \quad \cdot K_{m}(k \rho) \cos (m \theta)
\end{aligned}
$$

to rewrite the primary field as an expansion of Bessel functions and using the symmetry of the system

$$
\phi(\theta)=\phi(-\theta)
$$

and the limits

$$
\lim _{x \rightarrow 0} K_{n}(x)=\infty, \quad \lim _{x \rightarrow \infty} I_{n}(x)=\infty
$$

one finds the following general expression of the field

$$
\begin{gathered}
\varphi_{i}=\int_{-\infty}^{\infty}\left\{\sum _ { n = 0 } ^ { \infty } \left[a(n, k) I_{n}\left(k \rho^{*}\right)+\frac{I}{4 \pi^{2} \sigma_{\rho}}\left(2-\delta_{n}\right) K_{n}\left(k t^{*}\right)\right.\right. \\
\left.\left.\cdot I_{n}\left(k \rho^{*}\right)\right] \cos (n \theta)\right\} e^{i k z} d k \\
(\rho>t) \\
\varphi_{i}=\int_{-\infty}^{\infty}\left\{\sum _ { n = 0 } ^ { \infty } \left[a(n, k) I_{n}\left(k \rho^{*}\right)+\frac{I}{4 \pi^{2} \sigma_{\rho}}\left(2-\delta_{n}\right)\right.\right. \\
\varphi_{0}=\int_{-\infty}^{\infty}\left\{\sum_{n=0}^{\infty}\left[b(n, k) I_{n}(k \rho)+c(n, k) K_{n}(k \rho)\right] \cos (n \theta)\right\} \\
\varphi_{e}=\int_{-\infty}^{\infty}\left\{\sum_{n=0}^{\infty} d(n, k) K_{n}(k \rho) \cos (n \theta)\right\} e^{i k z} d k .
\end{gathered}
$$

Four boundary conditions can be applied at the surfaces of the two cylinders

$$
\begin{aligned}
& \left.\sigma_{\rho} \frac{\delta \varphi_{i}}{\delta \rho}\right|_{\rho=a}=\left.\left.\sigma_{o} \frac{\delta \varphi_{o}}{\delta \rho}\right|_{\rho=a} \quad \sigma_{\rho} \frac{\delta \varphi_{i}}{\delta \rho}\right|_{\rho=a}=\left.\sigma_{s}\left(\varphi_{i}-\varphi_{o}\right)\right|_{\rho=a} \\
& \left.\sigma_{o} \frac{\delta \varphi_{o}}{\delta \rho}\right|_{\rho=b}=\left.\left.\sigma_{e} \frac{\delta \varphi_{e}}{\delta \rho}\right|_{\rho=b} \quad \varphi_{o}\right|_{\rho=b}=\left.\varphi_{o}\right|_{\rho=b} .
\end{aligned}
$$

Using the orthogonality of the trigonometrical functions, the values for $a(n, k), b(n, k), c(n, k)$, and $d(n, k)$ now can be solved. Introducing

$$
\begin{aligned}
C_{1}(n, k)=k\{ & \sqrt{\sigma_{\rho} \sigma_{z}} I_{n}^{\prime}\left(k a^{*}\right)\left[k \sigma_{o} I_{n}^{\prime}(k a)-\sigma_{s} I_{n}(k a)\right] \\
& \left.+\sigma_{s} \sigma_{o} I_{n}\left(k a^{*}\right) I_{n}^{\prime}(k a)\right\}
\end{aligned}
$$

$C_{2}(n, k)=\left(\sigma_{o}-\sigma_{e}\right) K_{n}^{\prime}(k b) K_{n}(k b)$

$$
\begin{aligned}
& C_{3}(n, k)=k\left\{\sqrt{\sigma_{\rho} \sigma_{z}} I_{n}^{\prime}\left(k a^{*}\right)\left[k \sigma_{o} K_{n}^{\prime}(k a)-\sigma_{s} K_{n}(k a)\right]\right. \\
&\left.+\sigma_{s} \sigma_{o} I_{n}\left(k a^{*}\right) K_{n}^{\prime}(k a)\right\} \\
& C_{4}(n, k)=\sigma_{e} I_{n}(k b) K_{n}^{\prime}(k b)-\sigma_{o} I_{n}^{\prime}(k b) K_{n}(k b) \\
& C_{5}(n, k)=k\{ \sqrt{\sigma_{\rho} \sigma_{z}} K_{n}^{\prime}\left(k a^{*}\right)\left[k \sigma_{o} I_{n}^{\prime}(k a)-\sigma_{s} I_{n}(k a)\right] \\
&\left.+\sigma_{s} \sigma_{o} K_{n}\left(k a^{*}\right) I_{n}^{\prime}(k a)\right\}
\end{aligned}
$$

$C_{6}(n, k)=k\left\{\sqrt{\sigma_{\rho} \sigma_{z}} K_{n}^{\prime}\left(k a^{*}\right)\left[k \sigma_{o} K_{n}^{\prime}(k a)-\sigma_{s} K_{n}(k a)\right]\right.$

$$
\left.+\sigma_{s} \sigma_{o} K_{n}\left(k a^{*}\right) K_{n}^{\prime}(k a)\right\}
$$

$C_{7}(n, k)=k \sqrt{\sigma_{\rho} \sigma_{z}} \sigma_{s}\left\{I_{n}\left(k a^{*}\right) K_{n}^{\prime}\left(k a^{*}\right)-I_{n}^{\prime}\left(k a^{*}\right) K_{n}\left(k a^{*}\right)\right\}$

$C_{8}(n, k)=\sigma_{o}\left\{I_{n}^{\prime}(k b) K_{n}(k b)-I_{n}(k b) K_{n}^{\prime}(k b)\right\}$

$D(n, k)=4 \pi^{2} \sigma_{\rho}\left\{C_{1}(n, k) C_{2}(n, k)+C_{3}(n, k) C_{4}(n, k)\right\}$

$$
/ I_{n}\left(k t^{*}\right) \text {. }
$$


One finds

$$
\begin{aligned}
a(n, k)= & -\left\{C_{2}(n, k) C_{5}(n, k)+C_{4}(n, k) C_{6}(n, k)\right\} \\
& / D(n, k) \\
b(n, k)= & C_{2}(n, k) C_{7}(n, k) / D(n, k) \\
c(n, k)= & -C_{4}(n, k) C_{7}(n, k) / D(n, k) \\
d(n, k)= & -C_{7}(n, k) C_{8}(n, k) / D(n, k) .
\end{aligned}
$$

To compute the potential field, the integrals of (15) are discretised. After calculation of the coefficients of (18) and the Fourier coefficients of (15), the field is obtained using a fast Fourier transform.

It is not possible to calculate $a(n, 0)$ and $b(n, 0)$ straightforwardly because they contain terms that have infinite values for $k=0$. Such problems can be circumvented by using series expansions of $K_{n}(x)$ and $I_{n}(x)$ [9]. However, if the potential values are calculated along a line parallel to the axis, the terms $a(n, 0)$ and $b(n, 0)$ only influence the offset. In the numerical computations, these terms were given zero value, and after computation of the potential values an offset was added such that these values were zero at the largest negative and positive values of $z$, reflecting the fact that the potentials have to go to zero at infinity.

\section{REFERENCES}

[1] M. Brunner and U. T. Koch, "A precision multichannel cuff electrode optimized by simulation and experiment," J. Neurosci. Meth., vol. 35, pp. 93-106, 1990.

[2] B. M. Calancie and R. B. Stein, "Microneurography for the recording and selective stimulation of afferents: An assessment," Muscle Nerve, vol. 11, pp. 638-644, 1988.

[3] S. Y. Chiu, J. M. Ritchie, R. B. Rogart, and D. Stagg, "A quantitative description of membrane currents in rabbit myelinated nerve," $J$. Physiol., vol. 292, pp. 149-166, 1979.

[4] L. D. Clark, Jr. and D. J. Edell, "A probabilistic approach to the design of microelectrode layouts for neural signal transducers," in Proc. 11th Annu. IEEE Conf. Eng. in Med., Bio. Soc., 1989, pp. 1-2.

[5] N. Ganapathy and J. W. Clark, "Extracellular currents and potentials of the active myelinated nerve fiber," Biophys. J., vol. 52, pp. 749-761, 1987.

[6] T. M. Lefurge, E. V. Goodall, K. W. Horch, and A. S. Schoenberg, "Stability of chronic intrafascicular electrode recordings," in Proc. 11th Annu. IEEE Conf. Eng. in Med., Bio. Soc., 1989, pp. 932-933.

[7] B. K. Lichtenberg and C. J. Luca, "Distinguishability of functionally distinct evoked neuroelectric signals on the surface of a nerve," IEEE Trans. Biomed. Eng., vol. BME-26, pp. 228-237, 1979.

[8] W. B. Marks and G. E. Loeb, "Action currents, internodal potentials, and extracellular records of myelinated mammalian nerve fibers derived from node potentials," Biophys. J., vol. 16, pp. 655-668, 1976

[9] J. H. Meier, W. L. C. Rutten, A. E. Zoutman, H. B. K. Boom, and P. Bergveld, "Simulation of multipolar fiber selective neural stimulation using intrafascicular electrodes," IEEE Trans. Biomed. Eng., vol. 39, pp. 122-134, 1992.

[10] W. L. C. Rutten and J. H. Meier, "Selectivity of intraneural prosthetic interfaces for muscular control," Med. Biol. Eng., Comput., vol. 29, pp. NS3-NS7, 1991.
[11] W. L. C. Rutten, H. J. van Wier, and J. H. M. Put, "Sensitivity and selectivity of intraneural stimulation using a silicon electrode array," IEEE Trans. Biomed. Eng., vol. 38, pp. 192-198, 1991.

[12] U. Stanic, R. Acimović-anezic, N. Gros, A. Trnkoczy, T. Bajd, and M. Kljajic, "Multichannel electrical stimulation for correction of hemiplegic gait," Scand. J. Rehab. Med., vol. 10, pp. 75-92, 1978.

[13] R. B. Stein and M. N. Oguztöreli, "The radial decline of nerve impulses in a restricted cylindrical extracellular space," Biol. Cybern., vol. 28, pp. $159-165,1978$

[14] R. B. Stein, T. R. Nichols, J. Jhamandas, L. Davis, and D. Charles, "Stable long-term recordings from cat peripheral nerves," Brain Res., vol. 128, pp. 21-38, 1977

[15] D. Stephanova, N. Trayanova, A. Gydikov, and A. Kossev, "Extracellular potentials of a single myelinated nerve fiber in an unbounded volume conductor," Biol. Cybern., vol. 61, pp. 205-210, 1989.

[16] J. J. Struijk, J. Holsheimer, G. G. van der Heide, and H. B. K. Boom, "Recruitment of dorsal column fibers in spinal cord stimulation: Influence of collateral branching," IEEE Trans. Biomed. Eng., vol. 39, pp. 903-912, 1992.

[17] I. Tasaki, "A new measurement of action currents developed by single nodes of Ranvier," J. Neurophysiol., vol. 27, pp. 1199-1206, 1964.

[18] J. H. Meier, "Selectivity and design of neuro-electronic interfaces," Ph.D. thesis, Univ. Twente, Enschede, the Netherlands, 1992.

[19] W. L. C. Rutten, T. A. Frieswijk, J. P. A. Smit, T. H. Rozijn, and J. H. Meier, "3-D neuro-electronic interface devices for neuromuscular control: Design studies and realization steps," Biosensors, Bioelectron. vol. 10, pp. 141-153, 1995.

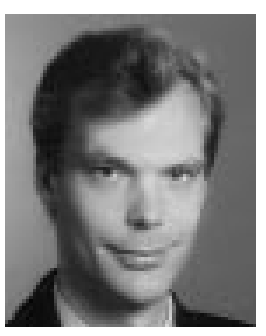

Jan H. Meier was born in Coevorden, the Netherlands, in 1962. He received the M.Sc. degree in physics in 1987 from the University of Groningen, Groningen, the Netherlands, and the Ph.D. degree in 1992 from the University of Twente, Enschede, the Netherlands. His Ph.D. research activities involved the modeling of neural stimulation and registration, the development of intraneural stimulation devices, and the testing of new stimulation strategies.

Since 1993, he has been working as Project Leader for the Biotronik GmbH \& Co., Erlangen, Germany. He is involved in the development of neurally controlled rateadaptive pacemakers as well as implantable neurostimulation systems.

Wim L. C. Rutten (M'96) was born in 1950. He received the Ph.D. in experimental physicis at Leiden University, Leiden, the Netherlands, in 1979. Thereafter, he studied the auditory system at the ENT Department of Leiden University Hospital.

Since 1985, he has been with the BMTI/Faculty of Electrical Engineering, University of Twente, as Assistant and Associate Professor of Biomedical Control Systems. His present research interests are neurotechnology (neuroelectronic interfaces, cultured probes), signal processing, and bioelectricity.

Herman B. K. Boom (A'89-SM'97) received the Ph.D. degree in 1971 in medical physics at the University of Utrecht, Utrecht, the Netherlands.

He joined the Department of Medical Physics and Medical Physiology at the same university, where he was engaged in the research of cardiac mechanics. Since 1976, he has occupied the Chair of Medical Electronics in the Faculty of Electrical Engineering, University of Twente, Enschede, the Netherlands. His research interests are cardiovascular system dynamics, bioelectricity, and rehabilitation technology. 